Modelling plastic deformation of metals over a wide range of strain rates using irreversible thermodynamics

This article has been downloaded from IOPscience. Please scroll down to see the full text article.

2009 IOP Conf. Ser.: Mater. Sci. Eng. 3012006

(http://iopscience.iop.org/1757-899X/3/1/012006)

View the table of contents for this issue, or go to the journal homepage for more

Download details:

IP Address: 131.180.130.109

The article was downloaded on 08/08/2011 at 10:08

Please note that terms and conditions apply. 


\title{
Modelling plastic deformation of metals over a wide range of strain rates using irreversible thermodynamics
}

\author{
Mingxin Huang ${ }^{1,2 *}$, Pedro E.J. Rivera-Díaz-del-Castillo ${ }^{1}$, Olivier Bouaziz ${ }^{2}$ and \\ Sybrand van der $\mathrm{Zwaag}^{1}$
}

(1) Faculty of Aerospace Engineering, Delft University of Technology, Kluyverweg 1, 2629 HS, Delft, The Netherlands

(2) ArcelorMittal Maizières, Research and Development, Voie Romaine-BP30320, 57283 Maizières-lès-Metz Cedex, France

*Corresponding author: mingxin.huang@arcelormittal.com

\begin{abstract}
Based on the theory of irreversible thermodynamics, the present work proposes a dislocation-based model to describe the plastic deformation of FCC metals over wide ranges of strain rates. The stress-strain behaviour and the evolution of the average dislocation density are derived. It is found that there is a transitional strain rate $\left(\sim 10^{4} \mathrm{~s}^{-1}\right)$ over which the phonon drag effects appear, resulting in a significant increase in the flow stress and the average dislocation density. The model is applied to pure $\mathrm{Cu}$ deformed at room temperature and at strain rates ranging from $10^{-5}$ to $10^{6} \mathrm{~s}^{-1}$ showing good agreement with experimental results.
\end{abstract}

\section{Introduction}

In general, models dealing with plastic deformation of metals are based on assumptions related to the processes of dislocation glide and accumulation, both of which are temperature and strain rate dependent. The classical Kocks-Mecking model [1] accurately describes the evolution of the average dislocation density and stress-strain behaviour in the low strain rate regime. However, at high strain rate regimes, the dislocation evolution and stress-strain behaviour follow different patterns. For instance, in metals and alloys such as copper [2], tantalum [3], aluminium [4] and stainless steels [5] when the strain rate exceeds about $10^{3}-10^{4} \mathrm{~s}^{-1}$ it has been found experimentally that the flow stress increases disproportionally. In order to quantitatively predict such stress-strain behaviour at high strain rates, constitutive dislocation-based models have been proposed such as the Zerilli-Armstrong model [6], the mechanical threshold stress model [2], the model incorporating phonon drag effects [5] and the model proposed by Meyers and co-workers [7].

The present work is an attempt to develop a dislocation-based model for both low and high strain rate deformation in FCC metals. The current formulation, which takes into account phonon drag effects, is an extension of the previous irreversible thermodynamics approach developed by the authors for low strain rate deformation [8]. 


\section{Model}

During plastic deformation in metals, generally three irreversible processes are considered to take place [9]: (1) dislocation generation, (2) dislocation annihilation (dynamic recovery) and (3) dislocation glide. According to the theory of irreversible thermodynamics, the entropy generation rate can be expressed as the sum of the products of generalised forces and their corresponding fluxes in the irreversible processes [10]. Therefore, the average entropy generation rate per unit volume due to the three irreversible processes is expressed as

$$
\frac{d_{i} S}{d t}=\underbrace{J_{1} X_{1}}_{\substack{\text { distocation } \\
\text { generation }}}+\underbrace{J_{2} X_{2}}_{\substack{\text { distocation } \\
\text { annihilation }}}+\underbrace{J_{3} X_{3}}_{\begin{array}{c}
\text { dislocation } \\
\text { glide }
\end{array}} .
$$

As a first order approximation, the generalised forces and their corresponding fluxes are assumed to take the following definitions. The general flux $J_{1}$ represents the average dislocation generation rate per unit volume defined as $J_{1}=\dot{\rho}^{+}$, where $\dot{\rho}^{+}$is the dislocation generation rate. The general force for dislocation generation or annihilation can be expressed as $X_{i}=E / T$, where $i=1$ for generation and $i=2$ for annihilation. $E$ is the average dislocation energy per unit length (including the average self energy and the average interaction energy) and is assumed to be $E=\mu b^{2} / 2$ where $b$ is the magnitude of the Burgers vector. $T$ is the absolute temperature. $J_{2}$ corresponds to the average dislocation annihilation rate per unit volume defined as $J_{2}=\dot{\rho}^{-}$, where $\dot{\rho}^{-}$is the dislocation annihilation rate (the total length of dislocations per unit volume annihilated during a time interval $d t$ ). $J_{3}$ is the general flux corresponding to dislocation glide expressed as $J_{3}=\rho_{m} v$, where $\rho_{m}$ is the average mobile dislocation density and $v$ is the average velocity of mobile dislocations. The relation between strain rate and mobile dislocation density is expressed by the well known Orowan relationship as $\dot{\gamma}=\rho_{m} v b$, where $\dot{\gamma}$ is the shear strain rate. $X_{3}$ is the general force for dislocation glide expressed as $X_{3}=\tau_{\text {eff }} b / T$, where $\tau_{\text {eff }}$ is the effective stress to move dislocations and it is assumed to be proportional to the shear flow stress $\tau$ as $\tau_{\text {eff }}=\kappa \tau$, where $\kappa$ is a constant and $0<\kappa<1$. Moreover, an increase of the average dislocation density $d \rho$ during the time interval $d t$ is the sum of the average dislocation generation and annihilation, i.e. $d \rho / d t=\dot{\rho}^{+}-\dot{\rho}^{-}$. Therefore, inserting all the above expressions of $J_{i}$ and $X_{i}(i=1,2,3)$ and $d \rho / d t=\dot{\rho}^{+}-\dot{\rho}^{-}$into equation (1), the entropy generation rate can be rewritten as

$$
\frac{d_{i} S}{d t}=\frac{1}{T}\left(\tau_{\text {eff }} \dot{\gamma}+\frac{\mu b^{2}}{2} \frac{d \rho}{d t}+\mu b^{2} \dot{\rho}^{-}\right) .
$$

Equation (2) is one way to express the relation between the entropy generation and the evolution of dislocation density. There is another way to link them, which will be shown as follow. The entropy generation rate is proportional to the energy dissipation rate and can therefore be expressed as $d_{i} S / d t=\left(d W_{\text {diss }} / d t\right) / T$ [10], where $d W_{\text {diss }}$ is the dissipative energy per unit volume transformed into heat during plastic deformation. Invoking the energy conservation law, the dissipative energy can be written as $d W_{d i s s} / d t=d W_{i n} / d t-d U_{s} / d t$, where $d W_{i n}$ is the input energy by loading during the time interval $d t$ and is expressed as $d W_{i n}=\tau d \gamma$, where $\gamma$ is the shear strain. $d U_{s}$ is the increase in stored energy due to the dislocation density increase (the sum of generation and annihilation) during $d t$ and is expressed as $d U_{s}=E d \rho$. Consequently, the second way to link the entropy generation and the evolution of dislocation is given by 


$$
\frac{d_{i} S}{d t}=\frac{1}{T}\left(\tau \dot{\gamma}-E \frac{d \rho}{d t}\right)
$$

Equations (2) and (3) are two different expressions to describe the entropy generation. Equating them offers an expression for the evolution of the average dislocation density:

$$
\frac{d \rho}{d t}=\frac{(1-\kappa) \tau \dot{\gamma}}{\mu b^{2}}-\dot{\rho}^{-}
$$

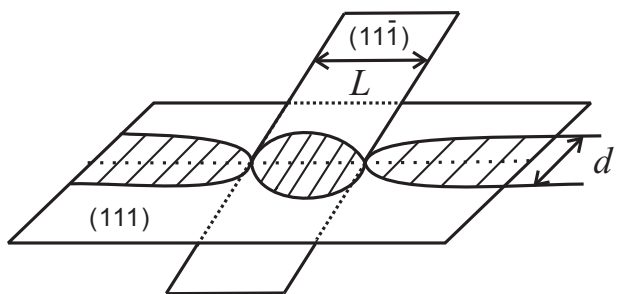

Figure 1. Schematic representation of cross-slip ((Friedel-Escaig model)

In equation (4), $(1-\kappa) \tau \dot{\gamma} /\left(\mu b^{2}\right)$ represents the dislocation accumulation rate, which implies that a small fraction of the input energy is stored in the material in the form of dislocation energy.

The current work deals with the room temperature deformation of FCC metals, where cross-slip is considered to be the dominant annihilation mechanism [11]. In FCC metals, the most common crossslip mechanism is compact cross slip (i.e. the Friedel-Escaig mechanism [12]) shown schematically in figure 1: the cross-slip segment $L$ immediately re-dissociates in the cross-slip plane (e.g. $(11 \overline{1})$ ) which is also a compact plane. More details about the modelling of the cross-slip mechanism may be found in the review paper by Puschl [11]. The dislocation annihilation process due to dislocation cross-slip can be expressed as [13]

$$
\dot{\rho}^{-}=N 2 L v_{c s} P_{c s}
$$

where $N$ is the number of sites per unit volume where such cross-slip events can occur and is approximately $N=\rho / L$ [13]. The factor 2 accounts for the fact that two screw dislocation segments with opposite sign of Burgers vector annihilate simultaneously at one single annihilation event. $v_{c s}$ is the attempt frequency for such cross-slip event and is taken to be $v_{c s}=(b / L) v_{D}$ [13] where $v_{D}$ is the Debye frequency $\left(v_{D}=10^{13} \mathrm{~s}^{-1}\right)$. The cross-slip length $L$ is related to its activation volume $V$ (figure 1) expressed as $L=V / d b$, where $d$ is the stacking fault width of screw dislocations which is determined by the stacking fault energy $\chi$ as $d=\mu b^{2} /(16 \pi \chi)$ [11]. $P_{c s}$ is the probability that the cross-slip attempt is successful and may be expressed as $P_{c s}=\exp [-\Delta G /(k T)]$ [13], where $k$ is the Boltzmann constant and $\Delta G$ is the activation energy for the cross-slip. The activation energy for cross-slip effectively increases with temperature but decreases with strain rate [1]; it may be expressed as $\Delta G=A k T \ln \left(v_{c s} / \dot{\gamma}\right)-\tau V k T /\left(\mu b^{3}\right)$, where $A$ is a constant in the order of 1 for a given temperature. The term $\tau V k T /\left(\mu b^{3}\right)$ accounts for the reduction in activation energy due to the applied stress; this is consistent with the well known Friedel-Escaig model [11, 12]. Thus, equation (5) can be rewritten as

$$
\dot{\rho}^{-}=\frac{\mu b^{4}}{8 \pi \chi V} v_{D} \exp \left[-A \ln \left(\frac{\mu b^{4}}{16 \pi \chi V} \frac{v_{D}}{\dot{\gamma}}\right)+\frac{\tau V}{\mu b^{3}}\right] \rho .
$$

The flow stress $\tau$ for pure FCC metals can be expressed as [5]

$$
\tau=\tau_{0}+\alpha \mu b \sqrt{\rho}+\tau_{\text {drag }}
$$

where $\tau_{0}$ is caused by the lattice friction which can be neglected for pure FCC metals. $\alpha \mu b \sqrt{\rho}$ 
accounts for the athermal friction stress due to other existing dislocations. $\alpha$ is a constant accounting for the interactions between dislocations and $\mu$ is the shear modulus. $\tau_{\text {drag }}$ is the friction stress due to viscous drag effects, which may be caused by phonons and/or electrons [9]. At moderate temperatures, phonon effects dominate over electron effects [9]. The contribution of electron effects can only be seen at very low temperatures (about 10 percent of Debye temperature) [9]. In the current work, such low temperature deformation is not considered so that electron effects are neglected. $\tau_{\text {drag }}$ is usually assumed to be expressed as $\tau_{d r a g}=B_{P H} v / b[5,9]$, where $B_{P H}$ is the phonon drag coefficient which is introduced by the so called phonon wind and fluttering mechanisms, i.e. $B_{P H}=\left\{B_{\text {wind }}^{0}+B_{\text {flutter }}^{0} /\left[1-\left(v / C_{t}\right)^{2}\right]\right\} T / \theta_{D}$ proposed by De Hosson et. al [14] where $\theta_{D}$ is the Debye temperature, $B_{\text {wind }}^{0}$ is a constant presenting the phonon wind mechanism at the Debye temperature, $B_{\text {flutter }}^{0}$ is a constant presenting the fluttering mechanism at the Debye temperature when the velocity of dislocation approaches 0 . Thus, the flow stress (equation (7)) can be rewritten as

$$
\tau=\alpha \mu b \sqrt{\rho}+\left[B_{\text {wind }}^{0}+\frac{B_{\text {fluter }}^{0}}{1-\left(\dot{\gamma} / b \rho_{m} C_{t}\right)^{2}}\right] \frac{T}{\theta_{D}} \frac{\dot{\gamma}}{b^{2} \rho_{m}} .
$$

Therefore, inserting equations (6) and (8) into equation (4) and substituting $\dot{\gamma}=d \gamma / d t$, the dislocation evolution can be rewritten as

$$
\begin{aligned}
& \frac{d \rho}{d \gamma}=\underbrace{\frac{(1-\kappa)}{\mu b^{2}}\left\{\alpha \mu b \sqrt{\rho}+\left[B_{\text {wind }}^{0}+\frac{B_{\text {flutter }}^{0}}{1-\left(\dot{\gamma} / b \rho_{m} C_{t}\right)^{2}}\right] \frac{T}{\theta_{D}} \frac{\dot{\gamma}}{b^{2} \rho_{m}}\right\}}_{I} \\
& -\frac{v_{D} \frac{\mu b^{4}}{\dot{\gamma}} \frac{v^{2} \pi V}{8 \pi \chi}\left[-A \ln \left(\frac{\mu b^{4}}{16 \pi \chi V} \frac{v_{D}}{\dot{\gamma}}\right)+\frac{\tau V}{\mu b^{3}}\right] \rho}{\underbrace{}_{I I}} .
\end{aligned}
$$

By solving equation (9) with an initial dislocation density $\rho_{0}$ before deformation, the dislocation evolution can be obtained. Then, the stress-strain behaviour can be predicted by utilizing equation (8).

The quasi-static deformation is assumed to be isothermal, while for high strain rate deformation (typically strain rates $>100 \mathrm{~s}^{-1}$ ) it is assumed to be adiabatic. The temperature increase $d T$ of the bulk during the strain interval $d \gamma$ for adiabatic deformation is related to the dissipative energy $d W_{d i s s}$ and is approximated as

$$
d T=\frac{d W_{d i s s}}{m_{\rho} C_{V}}=\frac{\tau d \gamma-E d \rho}{m_{\rho} C_{V}}
$$

where $m_{\rho}$ is the mass density and $C_{V}$ is the heat capacity. Here it is assumed that the heat flow in the bulk is fast enough such that no temperature gradients develop in the bulk. The temperature increase will decrease the shear modulus, increase the drag coefficient and increase the cross-slip rate.

\section{Results and discussion}

The model is applied to high purity oxygen free electronic $\mathrm{Cu}(99.99 \%)$ with a well annealed equiaxed grain structure and an average grain size of $40 \mu \mathrm{m}$ deformed at $295 \mathrm{~K}$. All experimental data in this work are taken from the literature by Follansbee and Kocks [2]. The grain size effects are not taken into account in the current model such that it can only be applied to coarse grained metals. The parameter values employed in the model are listed in table 1. Most of the values are obtained from the literature. The value for $B_{\text {wind }}^{0}$ used is smaller than the one (20.6 $\mu$-Pa.s) employed by De Hosson et. al 
[14]. $\rho_{m}, \rho_{0}, \kappa$, and $A$ are fitted according to the experimental data in figure 2 . It is noted that the stress, strain and strain rate in figures 2-5 are the normal stress $\sigma(\sigma=M \tau$ where $M$ is the Taylor factor), normal strain $\varepsilon(\varepsilon=\gamma / M)$ and normal strain rate $\dot{\varepsilon}(\dot{\varepsilon}=\dot{\gamma} / M)$, respectively.

\begin{tabular}{cccccccc}
\multicolumn{8}{c}{ Table 1 Values of parameters used in the model } \\
\hline$m_{\rho}\left(\mathrm{kg} / \mathrm{m}^{3}\right)$ & $C_{\mathrm{v}}(\mathrm{J} /(\mathrm{kg} \cdot \mathrm{K}))$ & $\mu(\mathrm{GPa})$ & $b(\mathrm{~nm})$ & $V\left(b^{3}\right)$ & $C_{t}\left(\mathrm{~m} \cdot \mathrm{s}^{-1}\right)$ & $\theta_{D}(\mathrm{~K})$ & $\alpha$ \\
$8940[15]$ & $386[15]$ & $47.4 \times \exp \left(-3.97 \times 10^{-4} T\right)[16]$ & $0.256[17]$ & $300[11]$ & $2.9 \times 10^{3}[14]$ & 343 & $0.4[8]$ \\
$B_{\text {wind }}^{0}(\mu \cdot \mathrm{Pa} \cdot \mathrm{s})$ & $B_{\text {flutter }}^{0}(\mu \cdot \mathrm{Pa} \cdot \mathrm{s})$ & $\rho_{m}\left(\mathrm{~m}^{-2}\right)$ & $\rho_{0}\left(\mathrm{~m}^{-2}\right)$ & $M$ & $\chi\left(\mathrm{J} \cdot \mathrm{m}^{-2}\right)$ & $\kappa$ & $A$ \\
$7[14]$ & $0.35[14]$ & $5 \times 10^{12}$ & $5 \times 10^{12}$ & $3.06[8]$ & $47 \times 10^{-3}[17]$ & $93.6 \%$ & 0.98 \\
\hline
\end{tabular}

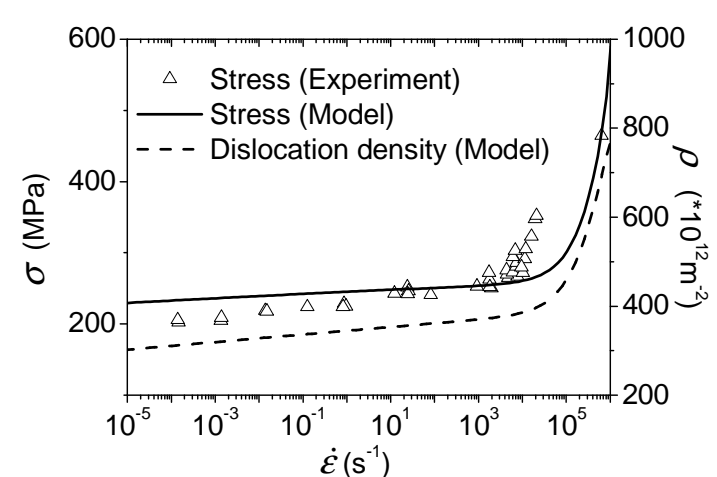

Figure 2. Flow stress and average dislocation density at $\varepsilon=0.15$ for wide ranges of stain rates

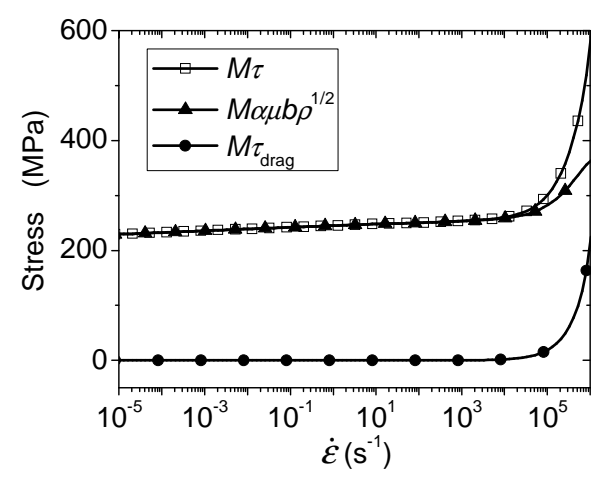

Figure 3. Stresses at $\varepsilon=0.15$ for wide ranges of stain rates

Figure 2 shows the flow stress at $\varepsilon=0.15$ for $\dot{\varepsilon}$ ranging from $10^{-5}$ to $10^{6} \mathrm{~s}^{-1}$. The model predictions are in agreement with experiments, demonstrating a transitional strain rate $\left(\square 10^{4} \mathrm{~s}^{-1}\right)$ beyond which the flow stress increases significantly with strain rate. The increase of the dislocation density also follows the same path. When the strain rate is higher than $\square 10^{4} \mathrm{~s}^{-1}$, i.e., when dislocation velocity is higher than 2-20 m/s (calculated by $v=M \dot{\varepsilon} \rho_{m} b$ ), the phonon drag effects appear, resulting in an increase of the flow stress (equation (8)) and the dislocation density (term $I$ in equation (9)). A comparable increase in dislocation density is also found by TEM in aluminium alloys deformed at strain rates ranging from $10^{3}$ to $6 \times 10^{3} \mathrm{~s}^{-1}$ [4]. It is noted that for pure $\mathrm{Cu}$ twinning may contribute to the plastic deformation at high strain rate [7]. However, as a first order approximation, the present work assumes that the dislocation glide is the main mechanism for plastic deformation and the contribution of twinning is neglected.

The flow stress $\sigma$ is comprised of dislocation contribution and phonon drag friction contribution (i.e., $\sigma=M \tau=M \alpha \mu b \sqrt{\rho}+M \tau_{\text {drag }}$ ). Their relative contributions are seen in figure 3. Both $M \alpha \mu b \sqrt{\rho}$ and $M \tau_{\text {drag }}$ increase sharply when $\dot{\varepsilon}$ is higher than $\sim 10^{4} \mathrm{~s}^{-1}$. But the increase of $M \tau_{\text {drag }}$ is more pronounced than $M \alpha \mu b \sqrt{\rho}$ at such strain rate regime. At low strain rate regime, only $M \alpha \mu b \sqrt{\rho}$ contributes to the flow stresses, while at high strain rate regime (higher than $\sim 10^{4} \mathrm{~s}^{-1}$ ) both $M \alpha \mu b \sqrt{\rho}$ and $M \tau_{\text {drag }}$ contribute to $\sigma$.

Figure 4 shows the stress-strain behaviour for three strain rates. The average dislocation density at the same deformation conditions are demonstrated in figure 5. It is clearly shown that both flow stress and dislocation density for the highest strain rate follow different profiles than their counterparts for the two lower strain rates. 


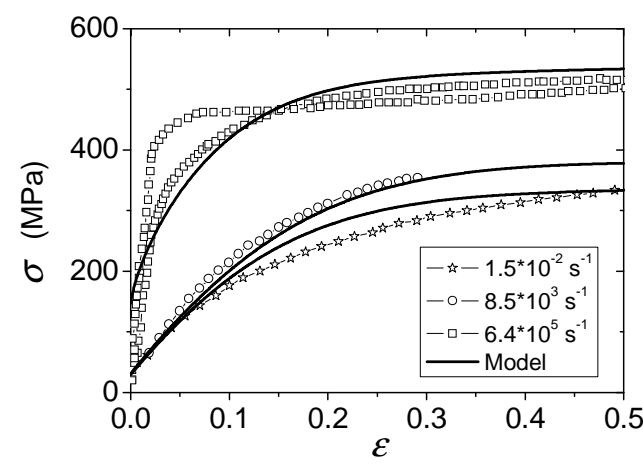

Figure 4. Model predictions (solid lines) and experimental data (symbols) for pure copper deformed at $295 \mathrm{~K}$ and at three strain rates (two experimental data sets for the highest strain rate).

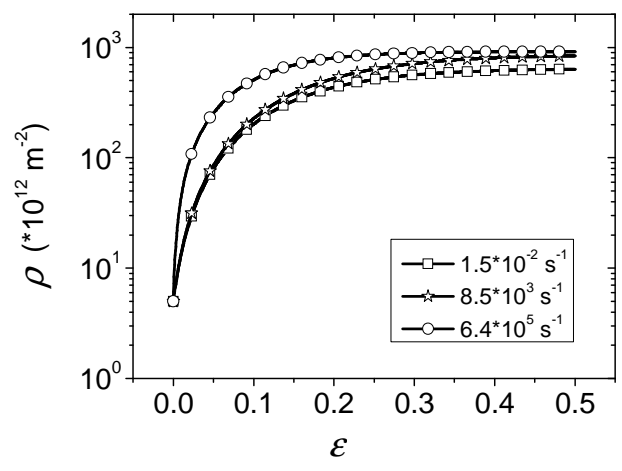

Figure 5. Model predictions of average dislocation density for pure copper deformed at $295 \mathrm{~K}$

\section{Summary}

A dislocation-based constitutive model has been proposed to describe the stress-strain behaviour and the evolution of the average dislocation for pure FCC metals. It is found that there is a transitional strain rate $\left(\sim 10^{4} \mathrm{~s}^{-1}\right)$ beyond which the phonon drag effects appear, resulting in a significant increase in flow stress and average dislocation density.

\section{Acknowledgments}

The authors employed at the TU Delft are grateful to ArcelorMittal Maizières for financial support. We acknowledge valuable comments by Professor Jeff T.M. De Hosson when developing the model.

\section{References}

[1] U. F. Kocks, H. Mecking, Progress in Materials Science 48 (2003) 171-273.

[2] P. S. Follansbee, U. F. Kocks, Acta Metallurgica 36 (1988) 81-93.

[3] R. Kapoor, S. Nemat-Nasser, Metallurgical and Materials Transactions A 31 (2000) 815-823.

[4] W.-S. Lee, T.-H. Chen, Scripta Materialia 54 (2006) 1463-1468.

[5] S. Nemat-Nasser, W.-G. Guo, D. P. Kihl, Journal of the Mechanics and Physics of Solids 49 (2001) 1823-1846.

[6] F. J. Zerilli, R. W. Armstrong, Journal of Applied Physics 61 (1987) 1816-1825.

[7] M. A. Meyers, D. J. Benson, O. Vohringer, B. K. Kad, Q. Xue, H. H. Fu, Materials Science and Engineering A 322 (2002) 194-216.

[8] M. Huang, P. E. J. Rivera-Díaz-del-Castillo, O. Bouaziz, S. van der Zwaag, Materials Science and Technology 24 (2008) 495-500.

[9] J. P. Hirth, J. Lothe, Theory of Dislocations, John Wiley \& Sons, New York, 1982.

[10] I. Prigogine, Introduction to Thermodynamics of Irreversible Process, John Wiley \& Sons, New York, 1961.

[11] W. Puschl, Progress in Materials Science 47 (2002) 415-461.

[12] J. Bonneville, B. Escaig, Acta Metallurgica 27 (1979) 1477-1486.

[13] W. D. Nix, J. C. Gibeling, D. A. Hughes, Metallurgical Transactions A 16 (1985) 2215-2226.

[14] J. T. M. De Hosson, A. Roos, E. D. Metselaar, Philosophical Magazine A 81 (2001) 1099-1120.

[15] A. Molinari, G. Ravichandran, Mechanics of Materials 37 (2005) 737-752.

[16] H. Mecking, B. Nicklas, N. Zarubova, U. F. Kocks, Acta Metallurgica 34 (1986) 527-535.

[17] E. Nes, Progress in Materials Science 41 (1997) 129-193. 